\title{
Parametric Resonance in the Drift Motion of an Ionic Bubble in near critical Ar Gas
}

\author{
A.F.Borghesanit and F.Tamburinit \\ Istituto Nazionale per la Fisica della Materia \\ Department of Physics "G.Galilei", via F. Marzolo, 8, I-35131 Padua, Italy
}

\begin{abstract}
The drift mobility of the $\mathrm{O}_{2}^{-}$ion in dense Argon gas near the liquid-vapor critical point has been measured as a function of the density. Near $T_{c}$ the zero-field density-normalized ion mobility $\mu_{0} N$ shows a deep minimum at a density smaller than $N_{c}$. This phenomenon was previously intepreted as the result of the formation of a correlated cluster of Argon atoms around the ion because of the strong electrostriction exerted by the ion on the highly polarizable and compressible gas. We now suggest that a possible alternative explanation is related to the onset of a parametric resonance of a bubble surrounding the ion. At resonance a large amount of energy is dissipated by sound waves in addition to viscous dissipation processes, resulting in the large mobility drop observed.
\end{abstract}

The transport properties of negative ions in dense rare gases and liquids have been recently subject of renewed interest because they can give information on how the microscopic structure of the fluid around the ion is modified by the ion-atom interaction [1,2]. Moreover, the possibility to greatly change with relative ease the gas density $N$ close to the critical point of the liquid-vapor transition gives the experimenters the unique opportunity to investigate the transition from the kinetic to the hydrodynamic transport regime [3].

The drift mobility of $\mathrm{O}_{2}^{-}$in dense $\mathrm{Ne}$ gas [2] or in liquid Xe [1] can be satisfactorily explained in terms of hydrodynamics if ions are assumed to be surrounded by a complex structure. The competition between short-range repulsive exchange forces between the weakly bound outer electron of the ion and the electrons of the atoms and the long-range polarization attraction of the ion with the atoms leads to the formation of a microcavity around the ion, which is in turn surrounded by a strong density enhancement [1.5].

A self-consistent field model has been developed to compute the structure shape [5]. The size of the complex is taken as effective hydrodynamic radius $R$ of the ion and its drift mobility is calculated by means of the Stokes formula $\mu_{0}=e / 6 \pi \eta R$, where $\eta$ is the viscosity. The agreement with $\mathrm{O}_{2}^{-}$mobility data in liquid Xe [4] is quite good, but the data in Ne gas near $T_{c}$ [2] are almost quantitatively reproduced only for $N>N_{c}$. In particular, the model does not reproduce even qualitatively the little dimple in the density-normalized mobility $\mu_{0} N$ for $N \approx 0.7 N_{c}$. In this Letter we show that this general feature of the mobility [6] can be explained by assuming that under certain termodynamic conditions a further dissipation mechanism becomes active in addition to viscous processes, namely sound wave emission by oscillations of the structure surrounding the ion.

Since the influence of electrostriction on the structure formation depends on the gas polarizability, we have carried out $\mathrm{O}_{2}^{-}$mobility measurements in Ar gas [7] because its polarizability is much larger than that of Ne. We used the pulsed photoinjection technique as for $\mathrm{Ne}[2]$ and $\mathrm{He}$ [3]. Details of the experiment are reported in literature 8.9. A small bunch of electrons is injected into the gas by irradiating a photocathode by means of a short UV light pulse. Electrons are captured by $\mathrm{O}_{2}$ impurities (in a concentration of $10-100$ p.p.m.) forming stable $\mathrm{O}_{2}^{-}$ions [10]. They drift under the action of an external electric field $E$ towards the anode inducing a detectable current. The analysis of its time dependence allows the determination of the drift time and, hence, of the drift velocity $v_{D}$ [11]. Finally, the mobility is calculated as $\mu=v_{D} / E$.

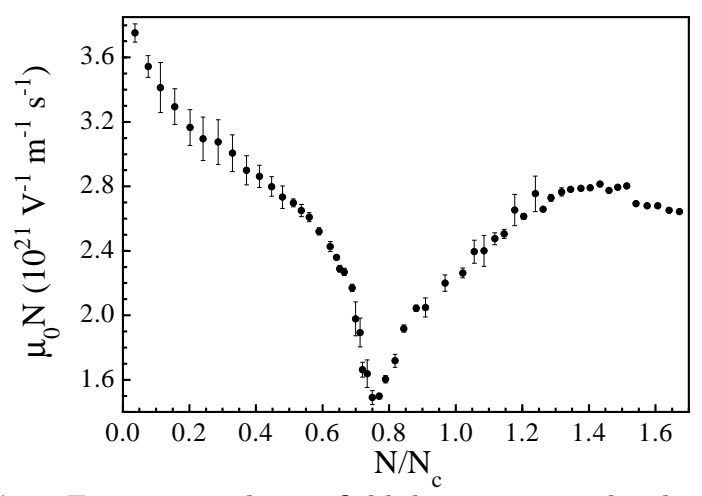

FIG. 1. Experimental zero-field density-normalized mobility $\mu_{0} N$ vs. $N / N_{c}$ for $T=151.5 \mathrm{~K}\left(\mathrm{~T} / \mathrm{T}_{\mathrm{c}} \approx 1.005\right)$.

In Fig.11 we show the measured zero-field densitynormalized mobility $\mu_{0} N$ vs. the reduced density $N / N_{c}$ for $T=151.5 \mathrm{~K}\left(\mathrm{~T} / \mathrm{T}_{\mathrm{c}} \approx 1.005\right)\left(N_{c}=8.08 \mathrm{atoms} \cdot \mathrm{nm}^{-3}\right.$ $\left.T_{c}=151.7 \mathrm{~K}\right) . \quad \mu_{0} N$ shows a very deep minimum for $N / N_{c} \approx 0.76$. The Stokes formula with constant radius reproduces the data, at most, for $N / N_{c} \geq 1.3$ [7]. The data are heuristically explained by assuming that a cluster of Ar atoms forms around the ion as a consequence of electrostriction [7]. This large structure interacts hydro- 
dynamically with the gas. The effective hydrodynamic radius depends on the local properties of the fluid. The good agreement with the data for $N / N_{c} \geq 0.5$ is obtained by introducing into the effective radius an adjustable contribution proportional to the size of the strongly correlated cluster. Thus, the hydrodynamic radius shows a complicated ad hoc density dependence, whose meaning is not easy to grasp. The reason might be that this model focuses only on the structure determined by the long-range part of the ion-atom interaction potential and does not take into account the presence of the microcavity closely surrounding the ion. Therefore, it neglects the possibility that the cavity may oscillate. In this case the energy of the moving complex, ion plus structure or, briefly, ionic bubble, can be dissipated by sound wave radiation in addition to viscous processes.

The present problem bears many affinities with that of the dynamics of electron bubble formation in liquid $\mathrm{He}$ [12], the adiabatic process where the repulsive electronatom interaction displaces a large number of atoms far away from the electron which is localized within the fluid dilation, or the electron solvation dynamics in water, where cavity contraction is induced by long-range attractive polarization interactions [13]. These problems have been treated in terms of a hydrodynamic model of cavity expansion [12] or collapse [13]. The dynamics of the cavity boundary is determined by the flow of the surrounding solvent. This is assumed to be spherically symmetric with velocity in the radial direction. In absence of energy dissipation and by assuming the incompressibility of the solvent, the cavity boundary velocity $U$ is described by the Rayleigh-Plesset (RP) equation

$$
\frac{\mathrm{d} U}{\mathrm{~d} t}=-\frac{3 U^{2}}{2 R}+\frac{1}{\rho R}\left[p(R)-p_{e}\right]
$$

where $R$ is the time dependent cavity radius, $\rho$ is the gas mass density, $p_{e}$ is the external pressure, and $p(R)$ is the pressure on the cavity boundary, expressed in terms of the free energy change $F$ in the process: $p(R)=$ $-(\partial F / \partial R) / 4 \pi R^{2}$. The equilibrium radius is obtained by minimizing $F$, but in absence of dissipation the system will not equilibrate. The cavity oscillates around the equilibrium value of the radius. Energy dissipation causes the damping of the oscillations. Within the given approximations, the first passage time, i.e., the time required for the cavity radius to expand (contract) from its initial value to its maximum (minimum) is taken as the lower bound for the relaxation timescale of the process. Viscosity and emission of sound waves drive the system towards the equilibrium [12,13].

It is obvious that also the drift motion of the ion is affected if energy is dissipated also by emission of sound waves. We therefore address the issue of the motion of the ionic bubble in dense Ar gas, considering the oscillations of the bubble boundary. We assume a spherical cavity with well-defined boundary of radius $R(t)$ with equilibrium value $R_{0}$. The main contribution to the restoring force is due to the surface tension. Although a gas has no surface tension, the enhanced density region around the cavity acts as a well-defined interface. Oscillations are initiated by collisions with energetic Ar atoms. Owing to the bubble smallness and to the asimmetry induced by the average motion driven by the external electric field, these collisions are not very frequent and occur in a spatially non homogeneous way. The equation of motion of the bubble boundary is then given by the RP equation, and, beside the impulsive nature of the driving force, is the same equation used for the bubble dynamics in the sonoluminescence experiments 15 .

So, for small distortion of the spherical shape a solution for the bubble radius is sought in the form $R_{0}+a_{n} Y_{n}$, where $Y_{n}$ is a spherical harmonic of degree $n$, and $a_{n}$ are the distortion amplitude coefficients. As bubble oscillations are initiated by collisions with energetic $\mathrm{Ar}$ atoms, this phenomenon can be basically described by a kicked rotator model, with the following equation of motion for the simple normalized periodic case: $a_{n}^{\prime \prime}+$ $\Gamma a_{n}^{\prime}-K f\left(a_{n}\right) \sum_{m=0}^{\infty} \delta(t-m \tau)=0$, where $m$ is an integer, $\Gamma$ the damping constant and $\tau$ the period between two kicks. Imposing a stochastic behaviour to the potential function, we can model collision events occurring with a gaussian distribution, e.g. in the quantum kicked rotator and electron localization problem [14. All properties of this equation are well known results of nonlinear dynamical system theory, which assure, under certain assumptions, the existence of a universal route to chaos. In the limit of small forcing the dynamics of the distortion amplitude can be cast in the form of the Mathieu-Hill $(\mathrm{MH})$ like equation 15

$$
b_{n}^{\prime \prime}+2 \xi_{m} b_{n}^{\prime}+\omega_{m}^{2}\left(1+\epsilon_{m} \cos 2 \tilde{t}\right) b_{n}=0
$$

with $b_{n} \propto R_{0}^{3 / 2} a_{n}(t) . \omega_{m}^{2}=\left(\omega_{0} / \omega\right)^{2}$ is the square of the ratio between the natural frequency of the bubble, $\omega_{0}$, and the excitation frequency $\omega$. The natural frequency is given by $\omega_{0}^{2}=\beta_{n} \sigma / \rho R_{0}^{3}$, where $\beta_{n}=(n-1)(n+1)(n+2)$, $\sigma$ is the surface tension, and $\rho$ is the mass density of the gas [15]. Primes denote differentiation with respect to the dimensionless time $\tilde{t}=\omega t$. Assuming that the bubble is empty, the surface tension can be calculated by using the parachoric formula [16] $\sigma=(P N)^{4}$, where $N$ is the gas number density and $P$ is a constant. For Argon $P \approx$ $1.39 \times 10^{-29} \mathrm{~J}^{1 / 4} \mathrm{~m}^{5 / 2}$. The term $\xi_{m}=2 n(n+2) \eta / \rho \omega R_{0}^{2}$ is a damping coefficient related to the viscosity $\eta$ whose values are found in literature [17]. Of the forcing term only the first Fourier component of amplitude $\epsilon_{m}$ has been retained.

For a dissipation-free system the Mathieu-Hill equation is known to give origin to parametric instability, when deviations from the spherical shape accumulate over many oscillation cycles. Floquet's theorem states 
that solutions of Eq.2 take the form [18]

$$
b_{n}(\tilde{t})=e^{\mu_{f} \tilde{t}} P_{n}(\tilde{t})
$$

where $\mu_{f}$ is the Floquet's index and $P_{n}$ is periodic. $\omega_{m}$ and $\epsilon_{m}$ span a plane geometrically divided into stability and instability regions 19]. We focus on the $n=2$ mode. In the instability regions $\mu_{f}^{2}>0$ and the envelope of the solutions to the $\mathrm{MH}$ equation grows exponentially. In the physical systems at hand this amplitude cannot grow indefinitely because dissipation stabilizes the surface dynamics. In the stability regions where $\operatorname{Re} \mu_{f}=0$ and $\operatorname{Im} \mu_{f}=2 \omega_{m}$ the solutions are periodic with angular frequency $\omega_{0}$ with a small modulation at angular frequency $2 \omega_{0}$. In this case the system experiences parametric resonance. In the limit of small forcing $\left(\epsilon_{m} \rightarrow 0\right)$, in absence of dissipation $\left(\xi_{m} \rightarrow 0\right)$ and close to the resonance $\left(\omega_{m} \approx 1\right)$, the non-zero solution for the Floquet's index is $\mu_{f} \approx 2 i \omega_{m}=2 i \omega_{0} / \omega$ [19]. We now show that $\operatorname{Im} \mu_{f}$ is related to the measured mobility $\mu_{0}$. From the expression of the bubble eigenfrequency, using the parachoric formula, we can write

$$
\omega_{m}=\frac{g}{\omega} N^{3 / 2}
$$

where $g=\left(N_{A} \beta_{n} P^{4} / M R_{0}^{3 / 2}\right)^{1 / 2}$ is a constant. $N_{A}$ is the Avogadro's number and $M$ is the atomic weight of Argon. $\omega$ is the excitation frequency. Therefore, under the hypotesis of collisional induced oscillations, $\omega=\omega_{\text {coll }} / l=2 \pi \nu_{\text {coll }} / l$, where $\nu_{\text {coll }}$ is the collision frequency and $l$ defines the order of a suitable subharmonic. In the Knudsen regime the mobility of a heavy ion of radius $R_{0}$ scattered off light particles of mass $m$ is

$$
\mu_{0} N=\frac{3 e}{8 R_{0}^{2} \sqrt{2 \pi m k_{\mathrm{B}} T}}
$$

and is related to the collision frequency by [1]

$$
\nu_{\text {coll }}=\frac{3 e N}{m\left(\mu_{0} N\right)}
$$

By inserting this result into Eq. 1 we get

$$
\operatorname{Im} \mu_{f} \propto \sqrt{N}\left(\mu_{0} N\right)
$$

where $\mu_{0} N$ is the experimentally measured zero-field density-normalized mobility. Therefore, $\sqrt{N} \mu_{0} N$ can be used to make a stability analysis of the bubble boundary motion.

In the real system, the ideal conditions of small forcing and absence of dissipation are not met and a parametric resonance is characterized by a minimum of the effective Floquet index, approximated by $\operatorname{Re} \mu_{f}$, which is shown to be related to $\sqrt{N} \mu_{0} N$. The minimum observed for $N / N_{c} \approx 0.76\left(N \approx 6.2\right.$ atoms $\left.\cdot \mathrm{nm}^{3}\right)$ is due to parametric resonance of the ionic bubble. We follow a heuristic line of reasoning. Once excited, the bubble starts oscillating with characteristic frequency $\operatorname{Im}\left\{\mu_{f}\right\}$ and growing amplitude given by $A(t)=\exp \left(\operatorname{Re}\left\{\mu_{f}\right\} t\right)$. The growth of the amplitude is limited because collision processes can absorb the oscillation energy. Therefore, if $\mathcal{T}$ is the time interval during which the bubble oscillates, $\operatorname{Re}\left\{\mu_{f}\right\} \mathcal{T} \simeq \mathcal{O}(1)$. On the other hand, according to calculations in the cavitation model [13], the oscillations last for a few cycles, hence $\operatorname{Im}\left\{\mu_{f}\right\} \mathcal{T} \simeq \mathcal{O}(1)$. Then, we have $\operatorname{Re}\left\{\mu_{f}\right\} \simeq \operatorname{Im}\left\{\mu_{f}\right\}$ and, owing to Eq.7, $\sqrt{N}\left(\mu_{0} N\right)$ is also related to the real part of the Floquet's index. The Ansatz must be then verified by a direct numerical stability analysis of Eq.2.

The minimum of $\mu_{0} N$ occurs at a density $N / N_{c} \approx 0.76$ where the resonance condition $\omega=\omega_{0}$ is met. In Fig.2 we plot vs. $N$ both $\omega_{0} \propto N^{3 / 2}$ and $\omega=\omega_{\text {coll }} / l \propto N$, approximately.

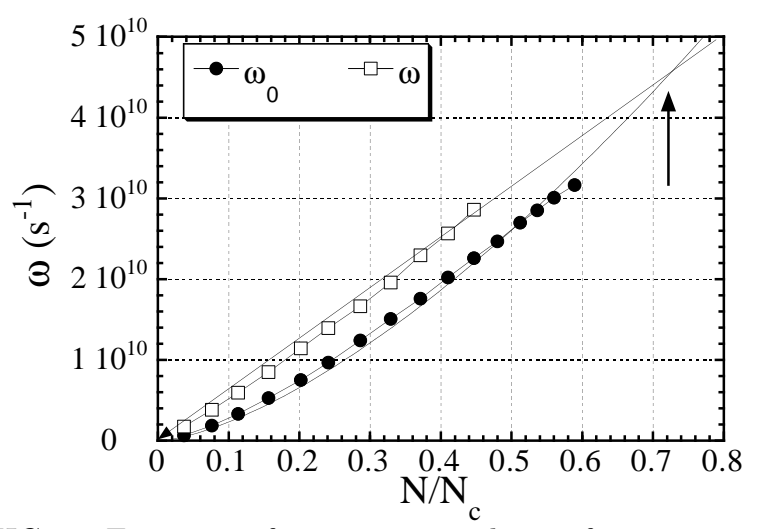

FIG. 2. Excitation frequency $\omega$ and eigenfrequency $\omega_{0}$ of the oscillating ionic bubble.

The two curves intersect for the right density value if $l \approx 2^{11}$. So, the excitation frequency must be $\approx 2^{11}$ times lower than the average collision frequency given by Eq.6. The factor $2^{11}$ can be explained assuming that only collisions with Ar atoms in the tail of the Maxwell-Boltzmann energy distribution function, namely those with average kinetic energy in excess of $3 k_{\mathrm{B}} T$, are energetic enough to initiate bubble oscillations. These energetic collisions are less frequent than average by the right factor.

We have numerically integrated the $\mathrm{MH}$ equation (Eq.2) by assuming an equilibrium radius $R_{0} \approx 10 \AA$, and have carried out the usual analysis of the stability bands. Usually, the Floquet's index is plotted vs. $\omega_{m}^{2}$. However, to compare the experimental data with the results of numerical analysis we have converted $\omega_{m}$ to $N$ by means of Eqns. 1 and 6 . In Fig. 3 we plot $\left(\mu_{0} N\right) \sqrt{N}$ and $\operatorname{Re} \mu_{f}$ as a function of the gas density $N$. The Floquet's index shows a number of small bands, but also a very deep minimum at the same $N$ of the experimental $\mu_{0} N$. This minimum is therefore associated with a strong para- 
metric resonance of the ionic bubble. The larger width of the experimental data can be explained by considering the fact that the experimental result is an average over a distribution of ionic bubble radii. At the condition of parametric resonance the amplitude of oscillation can reach substantial values and the kinetic energy gained by the external electric field can be efficiently dissipated by emission of sound waves.

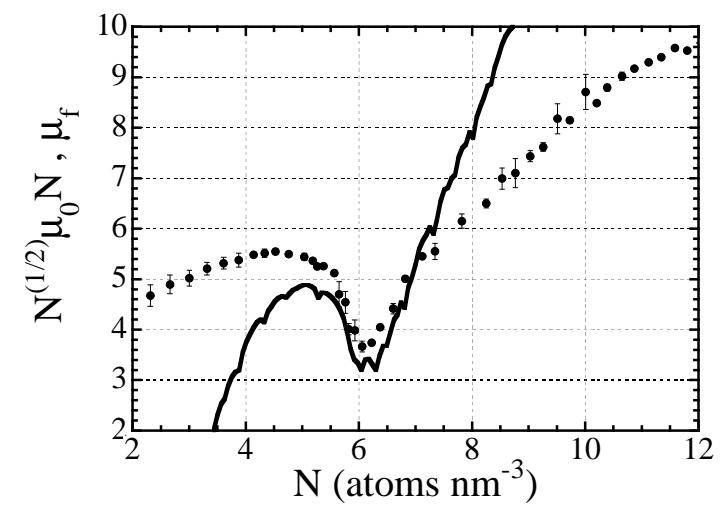

FIG. 3. Floquet's index $\mu_{f}$ obtained by numerical integrating the $\mathrm{MH}$ equation with $R_{0}=10 \AA$ and $\epsilon_{m}=1100$ compared to the experimental data $\mu_{0} N \sqrt{N}$ vs. $N / N_{c}$.

Close to the critical point the sound velocity $c$ is minimum $(\approx 190 \mathrm{~m} / \mathrm{s}[20])$ and sound dissipation is favoured. The sound intensity irradiated by an oscillating bubble in the long wavelength limit is [21] $I=2 \pi \rho c \omega^{2} R_{0}^{4}$. At the density of the minimum $\mu_{0} N$ the intensity irradiated per cycle is in the tens of meV range and a relevant part of the energy gained by the ion from the electric field is dissipated by means of this process in addition to the usual viscous processes. At higher $N$ the stiffness of the bubble surface becomes too large and the oscillations cannot be initiated so easily as in the resonance region. Hence, at high $N$ the contribution of the sound to energy dissipation is limited and $\mu_{0}$ is determined by the viscosity. This is why the high $-N$ region $\mu_{0} N$ can be well described by the Stokes formula. On the contrary, at much lower $N$ bubbles cannot form and $\mu_{0} N$ is determined by dissipation processes other than sound emission.

In the region where stable bubbles exist a further dissipation mechanism, though probably very small, should be considered. Within the bubble the ion undergoes a chaotic motion bouncing back and forth from the inner bubble wall. Molecular Dynamics studies [22] have shown a vibration of the ion within the cavity. The ion should therefore behave as an emitting antenna of characteristic frequency $\omega_{e} \approx 2 \pi v_{t h} / 2 R_{0}$, where $v_{t h}=\left(3 k_{\mathrm{B}} T / m_{i}\right)^{1 / 2}$ is the ion thermal velocity and $m_{i}$ its mass. At the experiment temperature $\omega_{e} \approx 10^{12} \mathrm{rads}^{-1}$. Moreover, this radiation should be modulated by the slower oscillation of the bubble boundary. We finally note that in this problem there is a moving interface between media of different polarizability, crossed by the strong electrical field of the ion. Therefore, an experiment could be designed to detect the expected quantum radiation emitted as a dynamic Casimir effect, that some authors consider the physical cause of sonoluminescence [23].

* e-mail address: borghesani@padova.infm.it

+ e-mail address: tamburini@iol.it

[1] K.F.Volykhin, A.G.Khrapak, and W.F.Schmidt, J.E.T.P. 81, 901 (1995)

[2] A.F.Borghesani, D.Neri, and M.Santini, Phys.Rev. E 48, 1379 (1993)

[3] A.F.Borghesani, F.Chiminello, D.Neri, and M.Santini, Int. J. Thermophys. 16, 1235 (1995)

[4] O.Hilt, W.F.Schmidt, and A.G.Khrapak, IEEE Trans. Dielect. Electr. Ins. 1, 648 (1994)

[5] A.G.Khrapak and K.F.Volykhin, J.E.T.P., 88, 320 (1999)

[6] R.Cantelli, I.Modena, and F.P.Ricci, Phys. Rev. 171 , 236 (1968)

[7] A.F.Borghesani, D.Neri, and A.Barbarotto, Chem. Phys. Lett. 267, 11 (1997)

[8] A.F.Borghesani, L.Bruschi, M.Santini, and G. Torzo, Phys. Rev. A 37, 4828 (1988)

[9] A.F.Borghesani and M.Santini, Phys. Rev. A 42, 7377 (1990)

[10] Dino Neri, A.F.Borghesani, and M.Santini, Phys. Rev. E 56, 2137 (1997)

[11] A.F.Borghesani and M.Santini, Meas. Sci. Technol. 1 , 939 (1990)

[12] M.Rosenblit and J.Jortner, Phys. Rev. Lett. 75, 4079 (1995)

[13] I.Rips, J. Chem. Phys. 106, 2702 (1997)

[14] S. Fishman, D.R. Grempel, and R.E. Prange, Phys. Rev. Lett. 49, 509, (1982);F.M. Izraelev and D.L. Shepelanskii, Theor. Math. Phys. 43, 417 (1980); H. G. Shuster, Deterministic Chaos, (VCH, Weinheim, 1988)

[15] M.P.Brenner, D.Lohse, and T.F.Dupont, Phys. Rev. Lett., 75, 954 (1995)

[16] J.O.Hirschfelder, C.F.Curtiss, and R.B.Bird, Molecular Theory of Gases and Liquids (Wiley, New York, 1964)

[17] N.J.Trappeniers, P.S. van der Gulik, and H. van den Hooff, Chem. Phys. Lett., 70, 438 (1980)

[18] E.L.Ince, Ordinary Differential Equations (Dover, New York, 1956)

[19] P.Bergè, Y.Pomeau, and C.Vidal, Order and Chaos (Wiley, New York, 1984)

[20] C. Tegeler, R. Span, and W. Wagner, VDI FortschrittBerichte, Reihe 3, Nr. 480, VDI Verlag, Düsseldorf (1997).

[21] L.D.Landau and E.M.Lifshitz, Fluid Mechanics, 74 (Pergamon, Oxford, 1987)

[22] E.L.Pollock and B.J.Alder, Phys. Rev. Lett. 41, 903 (1978)

[23] C.Eberlein, Phys. Rev. Lett. 76, 3842 (1996) 